\title{
A Study and Comparison of NIR Hyperspectral Imaging and other Emerging Biometric Techniques in Security System
}

\author{
Neeraj Pratap \\ Department of Computer Science \\ Gurukula Kangri Vishwavidyalaya, \\ Haridwar
}

\author{
Shwetank \\ Department of Computer Science \\ Gurukula Kangri Vishwavidyalaya, \\ Haridwar
}

\author{
Vikesh \\ Neelkanth Institute of Technology, \\ Meerut
}

\begin{abstract}
Different human body parts such as eyes, veins, voices, face, fingers etc. have been considered for the biometrics system. Even the typing style and signature of human being have been the part of biometrics. To satisfy the different needs of the global market, different numbers of biometric techniques have been considered with the several advantages as compared to the others technique. However, still today, no such technique able to completely satisfy the needs of current security system. Due to this the researchers continuously making their efforts and skills to produce the newer methods that will benefit a more to the security stage as compared to the traditional one. The use of hyperspectral imaging and the development of skin spectral curves has proved a vital significance in the security system. The objective of this paper is to study the hyperspectral imaging and other latest emerging biometric techniques for the security system.
\end{abstract}

\section{Keywords}

Biometrics, Emerging biometrics, Human recognition/ verification

\section{INTRODUCTION}

To satisfy the human requirement for different security issues, the biometric recognition of people is a growing research area and the continuous efforts are being made to develop the new techniques. The biometrics recognition refers to automatic security systems that depend on the physical or behavioral characteristics of an individual. Biometrics is considered as the most effective to satisfy the needs of the security systems and different constituent such as human body parts and personal characteristics, different imaging methods, have been used for biometrics systems. The researchers have shown their interest due to the automatic individual recognition/verification for the security applications. The person recognition can be classified by the two ways. On the one, person recognition refers to recognize the identity of a sample person using its biometric characteristics by selecting the best match or the $N$ most similar persons from given database[1,2].Generally such type of system are handled by a human expert who provides the final decision for the identity of the sample person. The human verification is concern with the automatic acceptance or rejection of an identity claimed. That is, a sample person claims an identity that is already stored in the system database and finally the acceptance or rejection is made by the system. The problem of person verification is very vast and in the last years many research groups and companies have shown their interest in the development of many verification techniques [3].

Face recognition/verification is one of the most effective biometric applications and has gained the significant consideration for last year's [4-6]. Different researchers from the various disciplines such as image processing, pattern recognition, neural networks, computer vision, computer graphics, and psychology have shown their interest in the problem of machine recognition of the human faces and developed a large number of algorithms in this regard. Still face recognition/verification remains an active subject of research due to several unsolved issue such as illumination variation, facial disguises, methods and type of image database used $[7,8]$.

Speaker recognition is another biometrics for which the researchers have shown their great efforts for many years [9].In Voice biometrics, the information, which are contained in the speech stream are used to perform identification. Voice biometrics generally benefit us by using good and higher quality microphones and several noise cancellation techniques but the several factors such as background and channel noise, variable and inferior microphones and telephones, extreme hoarseness, fatigue, or vocal stress[10] decrease the performance of these biometric.

The most general biometric is finger prints, based on the analysis of two-dimensional maps of finger prints produced by a number of different sensor types. For efficient storage, the ridge patterns on the finger are reduced to digital representation. These technologies are easy to implement but performance of these biometric are affected by many factors such as dryness, dirt or ageing [11, 12].

Although the current machine recognition system have gain a certain maturity level, but still their success is very limited due to the different conditions imposed on them [5]. Besides effectiveness, the major requirements for biometric systems are the availability and the affordability of biometric technologies.

Requirement for security systems are continuously increasing and the traditional biometric modalities are not supplying the needs of every possible security requirement [40]. So keeping in view all these facts, the different numerous emerging biometric modalities are presented, trying to minimize the limitations supported by the traditional biometrics. In this paper the latest biometric techniques are introduced. 


\section{CURRENT BIOMETRIC TECHNIQUES}

\subsection{Gait}

Gait is considered as a future biometric [13, 14] although it has been widely used from a long time. The individuals can be recognized by theirs walking pattern. Several methods have been proposed for the Gait biometric and some advance methods related to Gait are described here.

In 2005, Boyd and Little [15] described gait as "the coordinated cyclic combination of movements that result in human locomotion". The movements should occur with a specific temporal pattern. The foul gait cycle occurs in every cycle and this is generally a unique phenomenon. The different data types such as background substraction, silhouettes, optical flow and motion energy/history images are used in gait.

In 2003, Cunado D and Nixon MS [16] developed a system using joint trajectories, in which a hip joint trajectory is extracted from a sequence of images and the recognition is done on the basis of Fourier components of the trajectory.

In 2001, Boyd JE [17] developed a system based on pixel oscillation, in which the frequency of the gait and the timing of the component motions determine the frequency and phase of the pixel oscillations. A phasor, which represent a complex number, was used that represents a rotating vector, to represent the magnitude and phase of the oscillations at each pixel.

In 2008, Goffredo M [18] developed a technology based on markerless gait analysis. The technique generally follow the anthropometric proportions of human limps and the characteristics of the gait task. In this method single camera was used, not requires any camera calibration and also supports different styles of walking.

In 2008, Bouchrika I [19] study the gait identification, in which the effects of covariation on the recognition process are studied. The study also shows that's how these factors can affect the walking pattern. The study was conducted on a database of 440 samples and an accuracy of approximately $73.4 \%$ was achieved.

Gait presents advantages compared to other biometric modalities; still it is a subject of research for many years. It is still not widely used as a standalone application and generally proposed for multi-modal biometrics which increases the overall performance of the system.

\subsection{Thermal images / Thermogram}

In Traditional video cameras the light is reflected with sensors, so due to this the image intensities directly depend on the reflectivity of skin as well as external incident illumination, resulting the blurred reflectivity of skin. Thermal emission from the skin is the measurement, isolated from external illumination, under general conditions.

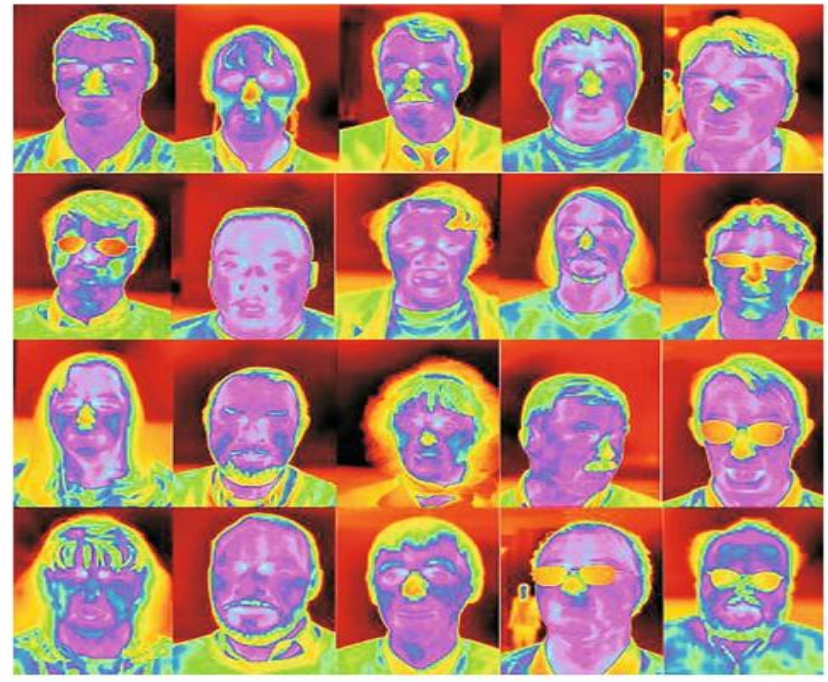

Fig 1: Thermal Images corresponding to the facial images

[20]

Different studies conducted by several researchers concluded that a pattern of unique heat distribution can be produced from the human face. This pattern can be analyzed and studied by using infrared cameras. The heat pattern, also called as "heat signature" of a human face is produced with bone densities, blood vessels and skin. The example of thermal images is the Equinox database [20] which is the collection of facial images in the different modalities. An example of Equinox image database is explored in Figure 1 [20].

Generally there are nine parameters, which are considered from the human face image for the construction of thermogram. Nose and ears are not considered as a parameter for the thermogram construction. When the face image is captured, its thermogram image is matched with the thermal graphs which are already stored corresponding to every image. The matching procedure is based on Monte Carlo analysis, which measures the performance. The Matching Performance is enhanced by considering the visible and thermal images together. In other words, the fusion of visible and thermal images increases the matching performance.

In 2003, Socolinsky DA conducted a study in which a matching performance comparison was shown of the two face recognition algorithms i.e. eigenfaces and Arena on to the Long-Wave Infrared and visible face image set [21]. The procedure was applied on the infrared videos of 91 subjects, in which the maximum classification performance was achieved $99 \%$ for ARENA on Long Wave Infrared (LWIR) Imagery while the minimum score achieved was $97 \%$ while the matching performance of eigen faces on LWIR imagery, was $96 \%$ and $87 \%$ for the same training sets.

In 2004 Buddharaju P, conducted a face recognition methodologies based on multiple appearance for which the input image was considered as the combination of visible and thermal images [22] and concluded that the thermal images produce the better result. The Recognition accuracy also increased when the same algorithm was used on to the combination of visible and thermal face images. The face recognition matching was performed by using Bayesian classifier on the Equinox database, resulting the higher matching rate $89.6 \%$. 
The main advantages of using thermal face images is that these face images can also perform the functioning in complete darkness, which show a great significance in covert surveillance. Thermal images also provide robustness to the different types of disguises such as the imaged that are beneath the skin and this makes their alteration almost impossible. The thermal images also not affected by the several types of skin diseases such as traumatic epidermic accidents. Although some limitations such as the presence of eye glasses reduce the performance because of low IR radiation due to glasses [23].

\subsection{Near infrared Multispectral/ \\ Hyperspectral Images}

The hyperspectral cameras are used to capture the Nearinfrared (NIR) images which provide a vast amount of information for human face recognition and which is not possible by the traditional cameras [24, 25].

In 2003, Zhihong P used the near-infrared hyperspectral images for face recognition, by using a database comprising of 200 subjects [25]. Then the spectral measurements were obtained for the several facial tissue types. The hyperspectral images were collected by using a CCD camera equipped with a liquid crystal tunable filter to provide 31 bands over the nearinfrared $(0.7-1.0 \mu \mathrm{m})$ as shown in Fig 2 [25].

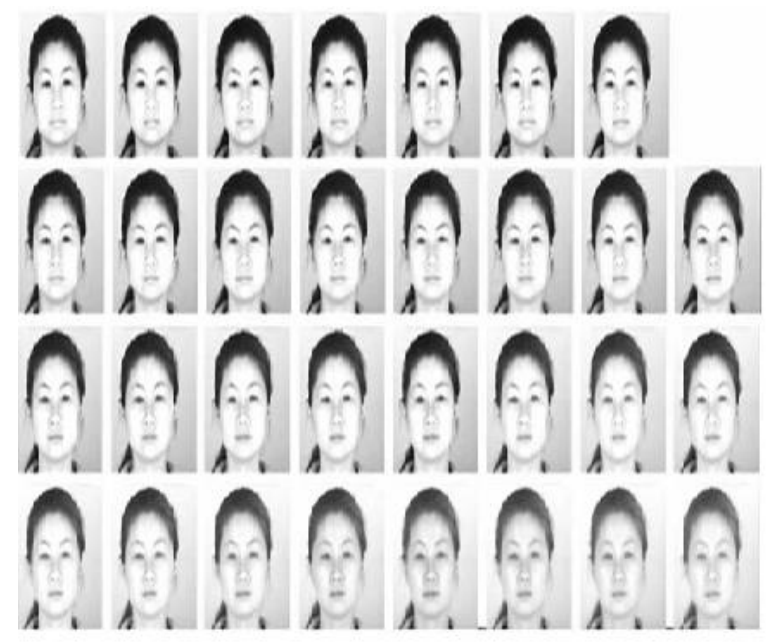

FIG 2 : An example of hyperspectral images [25]

A spectral curve in the near-infrared spectrum provides the sensing of subsurface tissue structure which varies from person to person, but relatively stable over time. The experimental results show that the local spectral properties of human tissue are not changed with the variant in face orientation and expression which allows hyperspectral information to be used for recognition over a large range of poses and expressions.

In multi dimensional face recognition system and data analysis, both the human skin information and its molecular composition (tissue, blood and structure etc) have the spectral information at many dedicated wavelength in EMS to discriminate the identical facial expression and features. In this progression, a hyperspectral face recognition system was developed in which three classes of methods, namely: WB (Whole Band) PCA, SBD (Single Band) PCA and BS-xFD (Band Subset Fusion Based) PCA are proposed for face recognition. The spectral measurements were limited over the hyperspectral frontal imagery database of 25 individuals with 33 spectral bands in visible range $(0.4-0.72 \mu \mathrm{m})$ [26].
In 2007, LI SZ and Chu RF provide another technique which includes active NIR imaging hardware, algorithms, and system design [27]. This technique solved the problem of illumination variation in face recognition. The methodology was obtained by extracting local binary pattern (LBP) features to compensate for the monotonic transform, resulting an illumination invariant face representation. To reduce the dimension and classification, the two algorithms LDA and Adaboost methods were developed. For the experiments 10000 face images of about 1000 people, all Chinese, were used for training the system and the accuracy was obtained by approximately $94.4 \%$.

Face recognition continues facing the challenges in reaching accurate results and still is wide area of research due to its limitations in accuracy. In a study an attractive approach is presented based on the image fusion of multiple imaging sources in Visible and NIR EMS. Hyperspectral data of 120 spectral bands is collected over hundreds of narrow contiguous light spectrum intervals within the Visible and Near Infrared Range (NIR). In this study the author investigated the efficiency of hyperspectral face recognition. The imagery was taken by the off the shelf sensor in both indoors and outdoors from various angles. Further spectral signatures are collected and used for feature extraction [28].

\subsection{Biometrics based on skin tissues properties}

In 2009, a new emerging biometric technology was described, which was based on the spectral properties of human skin [29]. Skin is the main organ of a human being which are made of multiple layers, various mixtures of biochemical substances and distinct structures, such as hair follicles, sweat glands and capillary beds. While every person has skin, but the characteristics of every individual skin is unique. The variation in human skin produced due to variation in thickness of skin layers, cell size and density within the skin layers, as well as in the chemical makeup of these layers.

The system hardware and software recognize skin differences and the optical affects they produce. The sensor illuminates a small patch of skin at multiple wavelengths of visible and near infrared light. The light which is reflected back, after being scattered in the skin, is measured for each of the wavelengths which are explored in Fig 3 . The light is passed to the skin and the changes are made in the light to extract an optical pattern, which are then compared to the pattern stored in the device to provide a biometric authorization.

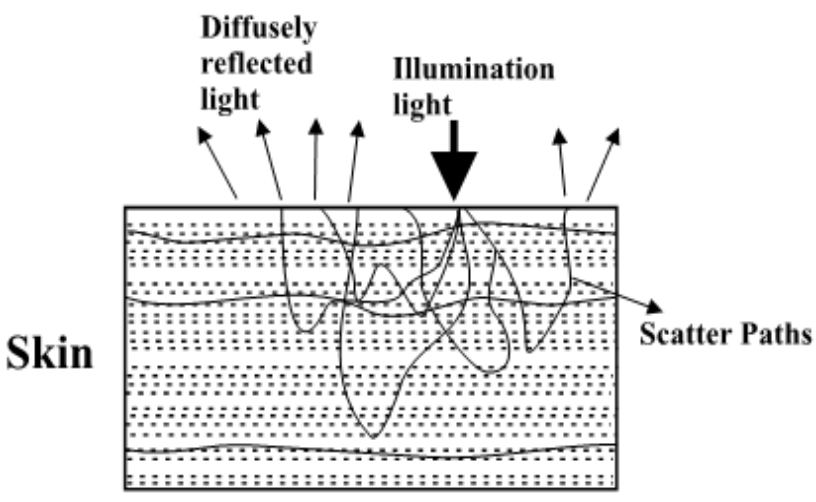

FIG 3: Radiation of light through skin [29]

The optical signal is affected by changes to the chemical and other properties of human being, which provides the confirmation about the sample to be a living human being 
tissue. In the similar way, the amputated tissue undergoes rapid changes in biochemistry; temperature and distribution of fluids within the various physiological compartments also change the optical signal. The changes in optical signal confirm that a sample authorized by the biometric sensor is a living human.

Spectroscopic approach removes the limitations of traditional technologies as a biometric. As the skin organ have a huge complexity in its, so it cannot be changed by synthetic materials used in parallel, liveness detection. In 2008, Davar P used the skin spectroscopy as a liveness detection solution for biometric systems [30].

\subsection{Finger-vein patterns}

In 2007, Miura $\mathrm{N}$ proposed a method for human identification [31], which was based on finger-vein patterns. The method was proposed as a scheme of biometric identification using biological information. The finger vein images were taken to obtain finger vein patterns which are obtained due to several facts such as irradiating the fingers with infrared rays, fluctuations in brightness due to variations in the light power or the thickness of the finger occur.

In the study conducted by several researchers, it was concluded that an image of a finger taken under infrared light contains not only the vein pattern, but also shading of the various finger bones and muscles. The proposed method extracts the centerlines of the veins from the image by estimating the curvature of the cross-sectional profile of the image and the vein pattern was obtained by analyzing all the profiles in a direction. The matching was done by the template matching method which is also previously used by the author in his earlier work $[32,33]$.

In this technique the mismatch ratio is generally greater especially in the cold weather due to the less visibility of the veins. So it is much required to investigate a device which can generate the vein pattern more clearly. The authors also studied another limitation i.e. the three dimensional rotation of the finger, which also decrease the matching performance. Another main disadvantage of this technology is that like fingerprints, finger vein cannot be easily fitted in small devices such as mobile phones, chip cards etc. "SecuaVeinAttestor" is a commercial product based on finger vein imaging. [34].

\subsection{Electrocardiogram (ECG) Pattern}

An ECG is the electrical recording of the heart and used in the investigation of heart diseases. ECG is widely used in clinic and has been used since the 20th century for the diagnosis of the different diseases related to the heart. According to different researchers, ECG is unique to every individual [3536]. In 2005, Israel SA et al proposed ECG as a biometric modality processing with quantifiable metrics [37]. The observed noise sources provide help in designing of the data filters. The uniqueness of an human being is characterized with the Fiducial points, calculated from the pattern.

The study show that the features extracted from the images are independent of sensor location, not affected by human being's state of anxiety, and unique to an individual. The sample experimental data were taken from both males and females ranges in the age of 22 to 48 . The individual data was recorded approximately seven two-minute tasks. The tasks where designed to stimulate different states of anxiety. The test was measured at two different points' neck and chest, and the researchers classified the heartbeats $82 \%$ and $72 \%$ for the two different points respectively, while in both cases, the identification or matching performance was $100 \%$ [37].

The experiment was carried to identify a population of individuals. Also the more data collection is being tried to test the scalability of the features on a large population and for also the stability of these features for a long time consideration.

\subsection{DNA}

DNA data are different from other standard biometrics in many ways. In it, a physical sample is taken for matching while in others an image or set of images are considered for matching. The matching is not performed in real-time and also it is not automatic. Generally in DNA matching the templates or feature extraction are not performed, rather the actual sample is compared [38].

During the matching, DNA is isolated and cut up into shorter fragments containing known areas and the fragments are sorted by size with the gel electrophoresis and then compared in many samples. An example of the identification that occurs with DNA method is described by Meeker-O'Connell [39]. The Performance Comparison of all these biometric modalities is shown with Table 1

Table-1: Performance Comparison of latest biometrics techniques

\begin{tabular}{|l|l|l|l|l|l|l|l|}
\hline \multicolumn{1}{|c|}{$\begin{array}{l}\text { Factors } \\
\text { Techniques }\end{array}$} & $\begin{array}{l}\text { Long Term } \\
\text { Stability }\end{array}$ & Performance & uniqueness & Acceptability & Universality & Cost & $\begin{array}{l}\text { Size } \\
\text { Sample }\end{array}$ \\
\hline Gait & Medium & Low & Medium & Medium & High & High & High \\
\hline Thermogram & Low & Medium & High & Medium & High & High & High \\
\hline $\begin{array}{l}\text { NIR Hyperspectral } \\
\text { images }\end{array}$ & High & High & High & High & High & High & High \\
\hline Skin spectroscopy & High & High & High & High & High & Medium & Medium \\
\hline $\begin{array}{l}\text { Finger-vein } \\
\text { patterns }\end{array}$ & High & High & High & Low & High & Medium & Medium \\
\hline ECG & Medium & Medium & High & High & High & Medium & Medium \\
\hline DNA & High & High & High & Low & High & Low & Low \\
\hline
\end{tabular}




\section{CONCLUSION}

In this paper the significance of hyperspectral imaging and other latest emerging biometrics technologies are presented. The human being contains a large number of body parts showing personal and behavioral characteristics and corresponding to these body parts different imaging methods have been suggested over the past years containing face, eyes, mouth, teeth, ears, hands, signatures, typing styles and others. Although most of the proposed techniques have gain a certain level of maturity but still a variety of unsolved problems still remain.

However, even in the most recent technologies, there are a lot of problems concerning the efficiency of each system but the hyperspectral imaging have shown a great significance and proved the much accuracy in identification process. Hyperspectral images provide much more relevant information about the subject as compared to the other imaging system. The skin tissue properties can also be used to produce the spectral signature which are not affected due to illumination and pose variation and remains same for a long time of period. Although researchers publish results that usually outperform their competitors, there is still no system except NIR hyperspectral imaging system that can guarantee reliably high performance for real security applications.

\section{REFERENCES}

[1] Jain AK, Flynn P, Ross AA. Handbook of biometrics, Springer, New York, 2007.

[2] Kotropoulos C, Tefas A, Pitas I, 2004, "Frontal face authentication using discriminating grids with morphological feature vectors". IEEE Transaction Multimedia Volume 2, pp. 14-26.

[3] Wayman JL, Jain AK, Maltoni D, Maio D, 2004, Biometric systems: technology, design and performance evaluation, Springer, New York.

[4] Jain AK, Li SZ, 2005, Handbook of face recognition. Springer, New York.

[5] Zhao W, Chellappa R, Rosenfeld A, P Phillips, 2000, "Face recognition: a literature survey", UMD Technical Report CAR- TR-948.

[6] Tefas A, Kotropoulos C, Pitas I, 2001, "Using support vector machines to enhance the performance of elastic graph matching for frontal face authentication", IEEE Transactions of Pattern Analysis and Machine Intelligence, Vol 23, pp. 735-746.

[7] Messer K, Kittler J, Sadeghi M, Marcel S, 2003, Face verification competition on the xm2vts database, in Proc. IAVBPA, Guildford, United Kingdom, 964-974.

[8] Messer K, Kittler J, Sadeghi M, Hamouz M, Kostin A, 2004, Face authentication test on the banca database, In Proc. ICPR04, Cambridge, United Kingdom, 523-532.

[9] Y Wang, LD Acero, 2005, Spoken language understanding, IEEE Signal Process Magazine, Volume 22, 16-31.

[10] JA Markowitz, 2000, Voice biometrics, In: Communications of the ACM, ACM, New York, Volume $43,66-73$.

[11] T Blackburn, M Butavicius, 1 Graves, D Hemming, 2002, Biometrics technology review. Australian department of Defense science and Technology Organisation.
[12] R Cappelli, D Maio, D Maltoni, 2006, Performance evaluation of fingerprint verification systems, IEEE Transactions of Pattern Analysis and Machine Intelligence, Vol 28, 3-18.

[13] MS Nixon, TN Tan, R Chellappa, 2005, Human identification based on gait, International series on biometrics. Springer, Berlin.

[14] MS Nixon, JN Carter, 2006, Human ID based on gait. In Proc. IEEE 94(11), 2013-2024.

[15] JE Boyd, JJ little, 2005, Biometric gait recognition. Lecture notes in computer science, Volume 3161. Springer, Berlin, pp. 19-42.

[16] D Cunado, MS Nixon, JN Carter, 2003, Automatic extraction and description of human gait models for recognition purposes, Journal of Computer Vision and Image Understanding, Volume 90,1-41.

[17] JE Boyd, 2001 , Video phase-locked loops in gait recognition. In Proc. of International conference on computer vision, Vancouver, BC, 696-703.

[18] M Goffredo, RD Seely, JN Carter, MS Nixon, 2008, Markerless view independent gait analysis with self-camera calibration. In Proc. IEEE international conference on automatic face and gesture recognition, Amsterdam, The Netherlands, 17-19.

[19] 1 Bouchrika, M Nixon, 2008, Exploratory factor analysis of gait recognition. In Proc. of $8^{\text {th }}$ IEEE international conference on automatic face and gesture recognition, Amsterdam, The Netherland.

[20] Equinox: Face database, equinox sensors. Com /products/ HID.html

[21] Socolinsky DA, Selinger A, Neuheisel JD, 2003, Face recognition with visible and thermal infrared imagery. Computer Vision Image Understanding, Volume 91, 72114.

[22] Buddharaju P, Pavlidis I, Kakadiaris I, 2004, Face recognition in the thermal infrared spectrum. In: CVPRW'04: Proceedings of the 2004 conference on computer vision and pattern recognition workshop (CVPRW'04), Volume 8. IEEE Computer Soc, Los Alamitos, p 133

[23] Pavlidis I, Tsiamyrtzis P, Manohar C, Buddharaju P, 2006, Biometrics: face recognition in thermal infrared. In: Biomedical eng handbook. CRC Press, Boca Raton

[24] Zhihong Pan MP, Healey G, Tromberg B, 2003, Face recognition in hyperspectral images. In: IEEE computer society conference on computer vision and pattern recognition (CVPR 2003), June, Madison, WI, USA, 334-339

[25] Zhihong Pan MP, Healey G, Prasad M, Tromberg B, 2003, Face recognition in hyperspectral images. IEEE Trans Pattern Anal Mach Intell 25:1552-1560

[26] Wei Di, Lei Zhang, David Zhang, and Quan Pan, November 2010, Studies on Hyperspectral Face Recognition in Visible Spectrum with Feature Band Selection, IEEE Transactions on Systems, Man, and Cybernetics - Part a: Systems and Humans, Volume 40, No.6, 1354-1361. 
[27] Li SZ, Chu R, Liao S, Zhang L, 2007, Illumination invariant face recognition using near-infrared images. IEEE Trans Pattern Anal Mach Intell, Volume 29, 627639.

[28] Stefan A. Robila, 2008, Towards Hyperspectral Face Recognition, Proc. SPIE 6812, Image Processing: Algorithms and Systems VI, Volume 6812.

[29] Lumidigm, 2009, http: // www. lumidigm. Com / index. html

[30] Davar P, 2008, Spectroscopically enhanced method and system for multi-factor biometric authentication. IEICE Trans Inf Syst E91-D(5):1369-1379

[31] Miura N, Nagasaka A, Miyatake T, 2007, Extraction of fingervein patterns using maximum curvature points in image profiles. The Institute of Electronics, Information and Communication Engineers, volume 90(8), 11851194

[32] Miura N, Nagasaka A, Miyatake T, 2004, Feature extraction of finger vein patterns based on iterative line tracking and its application to personal identification. System Computer Journal, Volume 35, 61-71.

[33] Miura N, Nagasaka A, Miyatake T, 2004, Feature extraction of finger-vein patterns based on repeated line tracking and its application to personal identification. Mach Vis Appl 15:194-203

[34] Hitachi, http: // www. hitachihec. co. jp / virsecur /secua_vein /vein01.htm

[35] Biel L, Pettersson O, Philipson L, Wide P, 2001, ECG analysis: A new approach in human identification. IEEE Transactions of Instrument Measurements, Volume $50,808-812$.

[36] Irvine $\mathrm{J}$, Wiederhold $\mathrm{B}$, Gavshon $\mathrm{L}$, Israel $\mathrm{S}$, McGehee S, Meyer R, Wiederhold M, 2001, Heart rate variability: a new biometric for human identification. In: International conference on artificial intelligence (ICAI'01), Las Vegas, Nevada, 1106-1111

[37] Israel SA et al, 2005, ECG to identify individuals. Pattern Recognition 38 (1):133-142.

[38] International Biometric Group, http: // www. biometricgroup. com/index.html

[39] Meeker-O'Connell A, How evidence works. How stuff works. evidence.htm http://www.howstuffworks.com/dna-

[40] Neeraj, Shwetank, Vikesh, 2014, "Classification of imagery data and face recognition techniques", IJCA, Volume 85(10), 21-26. 